bei nicht ischämischer Kardiomyopathie durchaus seine Berechtigung. Es bestehe deshalb zum jetzigen Zeitpunkt keine Veranlassung, DCM-Patienten den ICD grundsätzlich vorzuenthalten, zumal die Komplikationsrate (Hämatom, Infektionen) und das Risiko für eine inadäquate Schockabgabe Dank moderner Programmierungen (MA-
DIT-RIT) sehr niedrig, nämlich unter $2 \%$, liege. Es sei jedoch sinnvoll, vor allem bei älteren multimorbiden DCMPatienten die Indikation für einen ICD individuell zu stellen und die Möglichkeiten der modernen Herzinsuffizienztherapie voll auszuschöpfen. Dazu gehöre heute auch der Angiotensin-RezeptorNeprilysin-Inhibitor (ARNI).

\section{Jeder ICD-Schock verschlechtert die Prognose}

Der ICD ist für Patienten mit einer EF $\leq 35 \%$ lebensrettend. Doch jede Schockabgabe - ob adäquat oder nicht - führt nicht nur zu einer Beeinträchtigung der Lebensqualität, sondern auch zu einer myokardialen Schädigung, die die Prognose langfristig verschlechtert. Deshalb sind Strategien wichtig, die unnötige Schocks verhindern.

D ie primäre Frage bei einer dokumentierten ventrikulären Tachykardie sollte immer lauten: Braucht der Patient einen ICD oder nicht?", worauf Prof. Daniel Steven von der Kardiologischen Universitätsklinik in Köln auf der Hannover Herz Lungen Messe 2017 hinwies. Um diese Frage beantworten zu können, müsse man sich das EKG genau ansehen. Im Falle einer Ausflusstrakt-Tachykardie, die eine sehr gute Prognose habe, sei beispielweise ein ICD gar nicht indiziert. „Nicht jede VT erfordert einen ICD“, betonte Steven. Die sicherste Methode eine Schockabgabe zu verhindern sei, einen ICD erst gar nicht zu implantieren, also eine strenge Indikation zu stellen.

\section{Inadäquate Schocks sind} nicht selten

Sinn und Zweck des ICD ist es, im Falle von Kammerflimmern durch eine adäquate Schockabgabe den akuten Herztod zu verhindern. Aber es gibt Situationen - nämlich bei anhaltenden oder wiederkehrenden ventrikulären Tachykardien - in denen der Schock zwar notwendig ist, aber durch entsprechende Maßnahmen, die die VT beseitigen, überflüssig gemacht werden könnte. Dazu kommen inadäquate Schocks bei Sondendefekten und atrialen Arrhythmien. In allen ICD-Studien lag die Rate en inadäquaten Schocks bei fast $20 \%$ in 5 Jahren. „Häufigste Ursachen sind atriale Tachyarrhythmien bei Vorhofflim- mern und AV-junktionale Tachykardien“, berichtete Steven. Aber auch Sinustachykardien oder ein T-WellenOversensing können einen inadäquaten Schock auslösen.

\section{Verschiedene Mechanismen}

Jeder Schock, unabhängig davon, ob er notwendig ist oder nicht, verschlechtert

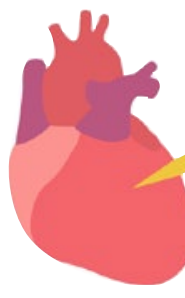
Lebensqualität beeinträchtigt, d. h. die physische, soziale, mentale und auch emotionale Befindlichkeit ist schlechter als bei Patienten ohne Schocks. „Dabei dürften unterschiedliche Mechanismen eine Rolle spielen“, erläuterte Steven. So führen die VTs selbst zu einer Kalziumüberladung der Zelle, was die Myokardfunktion des linken Ventrikels verschlechtert. Schocks verursachen kleine myokardialen Schädigungen, was wiederum Inflammation und Fibrosierung zur Folge hat. Zusätzlich dürfte auch eine Aktivierung der neurohumoralen Kaskade beteiligt sein.

\section{Ablation effektiver als Amiodaron}

Angesichts dieser ungünstigen Folgen einer Schockabgabe gilt der Grundsatz: Unnötige Schocks sollten verhindert werden. Inadäquate Schockabgaben durch supraventrikuläre Tachyarrhythmien samt Vorhofflimmern und AVdie Prognose. Darüber hinaus wird die
Ob eine optimale medikamentöse Therapie den ICD überflüssig mache, das müsse erst in entsprechenden Studien gezeigt werden. Solange solche nicht vorlägen, sollte an der Leitlinienempfehlung nichts geändert werden.

sti

Quelle: 5. Hannover Herz Lungen Messe,

10.3.2017 in Hannover junktionale Tachykardien können und sollen durch eine Katheterablation effektiv therapiert werden. Womöglich ist auch eine AVKnoten-Ablation erforderlich.

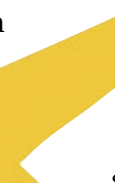
Gleiches gilt für VTs. Auch diese sollten soweit möglich abladiert werden. Dies ist sinnvoller als eine Amiodaron-Therapie bzw. eine Eskalierung der Amiodaron-Dosis. In einer kontrollierten Studie bei 259 Patienten mit ischämischer Kardiomyopathie konnte mit der Ablation im Vergleich zu eine antiarrhythmischen Medikation, vorwiegend Amiodaron, das rezidivfreie Überleben nach 4 Jahren um $28 \%$, bei Patienten, die bereits Amiodaron eingenommen hatten, sogar um $45 \%$ gesenkt werden. „Deshalb sollte in solchen Situationen eine frühzeitige Katheterablation der VT erwogen werden“, empfahl Steven.

Ansonsten empfiehlt sich eine „liberale“ Programmierung des ICD. In Studien (MADIT-RIT, ADVANCE III, EMPIRIC) ließ sich mit einem solchen innovativen Algorithmen die Rate unnötiger Schocks signifikant auf unter $2 \%$ senken, ohne dass die Mortalität ansteigt. Auch die Rate an synkopalen Ereignissen bzw. Krankenhausaufnahmen war nicht erhöht. „Solche modernen Programmierungen sind ungefährlich für den Patienten, aber im Hinblick auf die Verhinderung inadäquater Schockabgaben sehr effektiv“, schloss Steven.

sti

Quelle: 5. Hannover Herz Lungen Messe, 10.3.2017 in Hannover 